\title{
Dopaminergic control of attentional flexibility: inhibition of return is associated with the dopamine transporter gene (DAT1)
}

\author{
Lorenza S. Colzato ${ }^{1 *}$, Jay Pratt ${ }^{2}$ and Bernhard Hommel \\ 1 Institute for Psychological Research and Leiden Institute for Brain and Cognition, Leiden University, Leiden, Netherlands \\ 2 Department of Psychology, University of Toronto, Toronto, Canada
}

\author{
Edited by: \\ Anna C. Nobre, University of Oxford, \\ UK \\ Reviewed by: \\ Markus Ullsperger \\ Max Planck Institute for Neurological \\ Research, Germany \\ Anna C. Nobre \\ University of Oxford, UK \\ ${ }^{*}$ Correspondence: \\ Lorenza S. Colzato, Department of \\ Psychology, Cognitive Psychology Unit, \\ Leiden University, Postbus 9555, 2300 \\ RB Leiden, Netherlands. \\ e-mail: colzato@fsw.leidenuniv.nl
}

Genetic variability related to the dopamine (DA) transporter gene (DAT1) has received increasing attention as a possible modulator of human cognition. The 9-repeat allele of the DAT1 gene is presumably associated with higher striatal DA levels than the 10-repeat allele, which might support inhibitory control functions. We investigated the impact of the DAT1 gene on the inhibition of return (IOR) effect, which refers to the fact that people are slower to detect a target if it appears in a previously attended location. 140 healthy adults, genotyped for the DAT1 gene, performed an IOR task with stimulus-onset asynchronies (SOAs) between attention cue and target of 150-1200 ms. Nine-repeat carriers showed more pronounced IOR effect than 10/10 homozygous at short SOAs but both groups of subjects eventually reached the same magnitude of IOR. Our findings support the idea that striatal DA levels promote IOR, presumably by biasing the interplay between prefrontal and striatal networks towards greater cognitive flexibility.

Keywords: inhibition of return, dopamine transporter gene, dopamine, striatum, prefrontal cortex

\section{INTRODUCTION}

In the regulation of human cognition dopamine (DA) plays a key role, mainly through regulating the interplay between the prefrontal cortex (PFC) and the striatum. Some progress has been made in identifying genetic markers for the individual components of this interplay. For instance, the enzyme catechol-O-methyltransferase (COMT) is responsible for the degradation of DA in PFC (Karoum et al., 1994), while the DA transporter (DAT) is responsible for DA reuptake in the striatum (Sesack et al., 1998). Genetic variations in the dopamine transporter gene (DAT1) and the COMT gene (Val158Met polymorphism) have received increasing attention as possible modulators of cognitive control functions (Cools, 2008; Cools and D'Esposito, 2009).

The DAT1 gene is located on chromosome 5p15.3 (Giros et al., 1992; Vandenbergh et al., 1992) and contains a 40 base pair variable number of tandem repeats in the 30 -untranslated region ( $3^{\prime}$ UTRVNTR). Alleles from 3 to 13 repeats have been described, but the alleles with 9 and 10 repeats are the most frequently reported (Kang et al., 1999; Mitchell et al., 2000). The 9-repeat allele of the DAT1 gene has been associated with higher DA levels in the striatum relative to the 10-repeat allele. Recently, Bertolino et al. (2006) have observed similar effects on neuronal activity of the 10-repeat allele of the DAT1 gene and the Met allele of the COMT gene (associated with high prefrontal DA levels): the brain-activation patterns of subjects with putatively low striatal DA levels resembled that seen in subjects with putatively high prefrontal DA levels.

Cools (2008) and Cools and D'Esposito (2009), based on such reciprocal effects of prefrontal and striatal dopamine levels (and of corresponding effects of genes controlling such levels), have recently suggested that dopamine plays a key role in controlling the balance between cognitive stability on the one hand and cognitive flexibility on the other. The idea is that higher prefrontal DA levels promote cognitive stability by supporting working memory and increasing the resistance against distractors, whereas higher striatal DA levels promote cognitive flexibility by allowing the updating of novel relevant representations or the processing of goal-unrelated but nevertheless important information. Even though both stability and flexibility are important for proper cognitive functioning, shifting to one or the other extreme can be very counterproductive: too much stability would result in rigidity and too much flexibility in distractibility, either of which poses a major control dilemma (Goschke, 2000). Keeping stability and flexibility in proper balance, according to Cools and D'Esposito (2009), relies on continuous reciprocal adjustments of the prefrontal and striatal DA levels that are assumed to underlie these control functions. In terms of this assumed reciprocal relationship, 9-repeat carriers of the DAT1 gene with their higher striatal DA levels would be expected to be more biased towards the flexibility pole of the stability-flexibility dimension than 10-repeat carriers would be.

In the present study, we tested this hypothesis by applying it to the inhibition of return (IOR) effect (Posner and Cohen, 1984), a particularly reliable phenomenon in human attention that has been hypothesized to promote flexibility. It is observed when people attend sequential displays or scan complex visual scenes (Klein, 1988), or in other circumstances where they move their attentional focus from one object to another until an interesting or searched-for object has been found. As Posner and Cohen (1984) have shown, cuing the location of a visual target by means of a randomly varying, peripheral stimulus (e.g., a flash at the location where the target will occur) can improve performance at very short stimulus-onset asynchronies (SOAs) but impairs performance at longer SOAs. Once a given location has been inspected and 
attention has moved to another location, the time needed to return to that previous location is increased - presumably to enhance the efficiency of attentional scanning by biasing it away from irrelevant, old information and to prepare the system for the intake of novel information (Klein, 1988).

Considering that the DAT1 gene is associated with DA availability in the striatum (provided by the substantia nigra), there are a number of reasons suggesting that DAT1 might impact IOR. IOR is enhanced after the intake of d-amphetamine (Fillmore et al., 2005), and is reduced in Parkinson's patients and Huntington's patients, who suffer from a loss of nigrostriatal dopaminergic cells and of a bilateral neural degeneration of the striatum, respectively (Filoteo et al., 1997; Yamaguchi and Kobayashi, 1998; Couette et al., 2008). These studies fit with the proposed crucial role of dopamine as neurobiological mechanism underlying IOR (Poliakoff et al., 2003). Moreover, Colzato and Hommel (2009) found that in contrast to cocaine-free controls, recreational cocaine users do not show a reliable IOR. At long term, chronic use of cocaine is associated with reduced functioning of dopaminergic D2 (DAD2) receptors (Volkow et al., 1999), which are involved in regulating the functional expression of DAT1 (Meiergerd et al., 1993; Dickinson et al., 1999; Mortensen and Amara, 2003).

The goal of the present study was thus to examine whether individual differences related to the DAT1 polymorphism can predict the magnitude and/or temporal characteristics of the IOR effect. Given that previous studies showed reduced IOR in populations affected by reduced striatal DA activity, such as cocaine users, Parkinson's and Huntington's patients (Filoteo et al., 1997; Yamaguchi and Kobayashi, 1998; Couette et al., 2008; Colzato and Hommel, 2009), we expected 10/10 homozygous carriers (presumably combining relatively low striatal and relatively high prefrontal DA levels) to show a smaller IOR than 9-repeat carriers (presumably combining relatively high striatal and relatively low prefrontal DA levels). In the sense of Cools and D'Esposito (2009), we thus expected 9-repeat carriers to show more evidence of attentional flexibility, as expressed by more IOR.

Although the prediction of the main effect of DAT1 on IOR is straightforward, the impact of the gene on the time course of IOR is more difficult to predict. There are at least two, not mutually exclusive possibilities to consider. On the one hand, the higher striatal DA levels in 9-repeat carriers may allow for more or stronger inhibition, which would imply that the maximum magnitude IOR reached by these individuals would be higher than in 10/10 homozygous carriers. Alternatively, the combination of lower prefrontal and higher striatal DA levels in 9-repeat carriers may speed up the processes responsible for the behavioral expression of IOR without necessarily leading to a stronger effect. IOR is commonly assumed to reflect the net result of two concurrent processes: a facilitatory effect that quickly decays (presumably a spillover of cue-induced neural activity; cf., Fecteau and Munoz, 2005) and the build-up of a longer-lasting inhibitory effect. The size of IOR at any given moment is thus the result of the current relative strength of the facilitatory and the inhibitory process - with the former being more dominant at short SOAs (so that, on average, performance is better if cue and target appear at the same location) and the latter becoming more dominant (and performance being better if cue and target appear at different locations) as SOA increases (Posner and
Cohen, 1984). DAT1 might affect the time course resulting from this interplay between facilitation and inhibition, be it by shortening the duration of the facilitatory component (e.g., by accelerating the attentional disengagement from the cue; cf., Lupianez et al., 2004) and/or by leading to an earlier onset of the inhibitory component. In any case, the relative strength of the inhibitory process would be greater in 9-repeat carriers. If so, the transition from facilitation to inhibition would be faster in these individuals, so that the maximum IOR would be reached after a shorter SOA in 9-repeat than in 10-repeat carriers. Obviously, these two possibilities do not necessarily exclude each other, so that 9-repeat carriers may show both a faster build-up of net IOR and a higher maximum.

In a previous study of ours on the relationship between COMT and task switching (Colzato et al., 2009), we observed a pattern that fits better with the second option: Val/Val and Met/ - carriers showed comparable task-switching costs and longer preparation intervals, whereas Val/Val carriers (which are assumed to have lower prefrontal and higher striatal DA levels) were faster to switch at short intervals. Apparently, genetic differences affected efficiency and speed rather than maximum performance in this particular case. However, with respect to DAT1 effects, the available evidence does not allow preferring one possibility over the other. We therefore studied the time course of IOR over a wider interval, ranging from 150 to $1200 \mathrm{~ms}$, to see whether 9-repeat carriers would produce a higher maximum IOR or just reach that maximum earlier than $10 / 10$ homozygous carriers.

\section{MATERIALS AND METHODS PARTICIPANTS}

Hundred-forty-four young Caucasian healthy adults (67 male/73 female), with a mean age of 21.9 years $(\mathrm{SD}=2.6$, range 18-30) and 115.5 IQ (SD = 3.1, range 100-130); served as participants for partial fulfillment of course credit or a financial reward. The sample was drawn from adults in the Leiden and Rotterdam metropolitan area (The Netherlands), who volunteered to participate in studies of behavioral genetics. Exclusion criteria were any major medical illness that could affect brain function, current and/or past substance abuse, neurological conditions, history of head injury, and personal history of psychiatric medical treatment. Participants were selected via a phone interview using the mini international neuropsychiatric interview (MINI; Lecrubier et al., 1997). The MINI is a well established brief diagnostic tool in clinical and stress research (Sheehan et al., 1998; Elzinga et al., 2007; Elzinga et al., 2008) that screens for several psychiatric disorders including schizophrenia, depression, mania, ADHD, and obsessive-compulsive disorder. Written informed consent was obtained from all participants after the nature of the study was explained to them; the protocol was approved by the ethical committee of the Department of Psychology at Leiden University.

\section{APPARATUS, STIMULI, AND TASK}

The experiment was controlled by a PC attached to a 17 -inch monitor with a refresh rate of $100 \mathrm{~Hz}$. The task was modeled after Castel et al. (2003). The experiment took place in a dimly illuminated, sound attenuated room. Participants were seated $45 \mathrm{~cm}$ in front of a computer monitor. They were asked to fixate on a central cross $\left(0.1^{\circ} 30.1^{\circ}\right)$ and to make no eye movements during the experimental trials. 
The sequence of events is shown in Figure 1. All stimuli were presented in white $\left(77.0 \mathrm{~cd} / \mathrm{m}^{2}\right)$ on a black background $(0.5 \mathrm{~cd} /$ $\mathrm{m}^{2}$ ). The initial display was presented for $1000 \mathrm{~ms}$ and consisted of two placeholder boxes located on the horizontal meridian to the left and right of the fixation point. The boxes were centered 58 from the fixation point and were 18 square. One of the boxes was then cued by outlining the perimeter for $50 \mathrm{~ms}$. One of five randomly determined SOAs then followed the onset of the cue $(50,250,750,1000,1500 \mathrm{~ms})$. After the variable SOA, a target circle $(0.78 \mathrm{~cm})$ appeared in one of the two boxes (on $80 \%$ of the trials; the remaining $20 \%$ served as catch trials in which no target was presented). Participants were asked to respond to the target as quickly and as accurately as possible by pressing the space bar of the computer keyboard (regardless of the location of the target) and to remain fixated throughout each trial. The next trial began $500 \mathrm{~ms}$ later. The experiment consisted of 300 trials with cues and targets being equally likely to occur at the left and right locations.

\section{DNA LABORATORY ANALYSIS}

Genomic DNA was extracted from saliva samples using the Oragene $^{\mathrm{TM}}$ DNA self-collection kit following the manufacturer's instructions (DNA Genotek, Inc., 2006).

The DAT1 polymorphism was amplified on an MJ DNA engine thermal cycler (MJ Research) with an initial denaturation at $94^{\circ} \mathrm{C}$ for $4 \mathrm{~min}$, followed by 32 cycles of $45 \mathrm{~s}$ at $94^{\circ} \mathrm{C}, 45 \mathrm{~s}$ at $68^{\circ} \mathrm{C}$, and $60 \mathrm{~s}$ at $72^{\circ} \mathrm{C}$, and a final elongation of $5 \mathrm{~min}$ at $72^{\circ} \mathrm{C}$. The $25-\mathrm{ml}$ reaction mixture consisted of $50 \mathrm{mM}$ Tris ( $\mathrm{pH}$ 9.0), $20 \mathrm{mM} \mathrm{NH}_{4} \mathrm{SO}_{4}, 3 \mathrm{mM}$ $\mathrm{MgCl}_{2}, 200 \mathrm{mM}$ dNTPs, $0.5 \mathrm{mM}$ primers, and $1 \mathrm{U}$ Taq polymerase (Invitrogen). Products were electrophoresed on 2\% agarose gel and visualized with ethidium bromide. The oligo primer sequences used

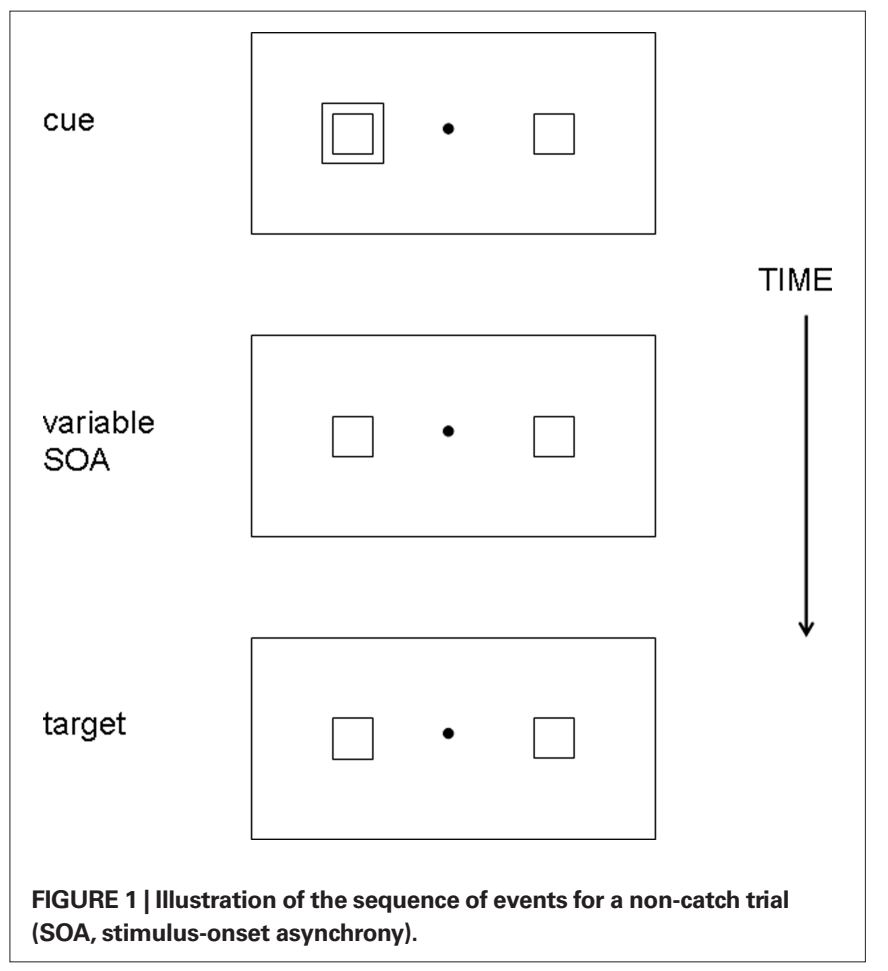

to amplify the VNTR are DAT1-F: 5'-TgT ggT gTA ggg AAC ggC CTg Ag-3' DAT1-R: 5'-CTT CCT ggA ggT CAC ggC TCA Agg, as originally described in Waldman et al. (1998). Each individual was genotyped twice.

DNA samples were unobtainable from four participants and these adults were excluded from any further analysis.

\section{PROCEDURE AND DESIGN}

All participants were tested individually. Individual IQs were determined by means of a 30-min reasoning-based intelligence test (SPM). The SPM assesses the individual's ability to create perceptual relations and to reason by analogy independent of language and formal schooling; it is a standard, widely used test to measure Spearman's g factor and of fluid intelligence in particular (Raven et al., 1988). Participants provided completed the SPM and subsequently performed on the behavioral task measuring IOR. The behavioral task took about 20-min. Participants were allowed to take a short break (maximal $5 \mathrm{~min}$ ) between task blocks.

\section{STATISTICAL ANALYSIS}

Independent samples $t$-tests were performed for analysis of age, sex, IQ differences between 10/10 homozygous and 9-repeat carriers. The effect of DAT1 genotype on IOR was assessed by means of $2 \times 2 \times 5$-ANOVAs with DAT1 (10/10 homozygous vs. 9-repeat carriers) as between-subject factor, and Trial Type (i.e., cued vs. uncued) and SOA (5 SOAs) as within-subject factor. We adopted a significance level of $p<0.05$ for all statistical tests.

\section{RESULTS}

The genotype distribution of the DAT1 polymorphism in our population was $8210 / 10$ homozygous subjects (58.35\%) and 58 9-repeat carriers subjects $(41.65 \%)$. The allelic distribution of the gene was in Hardy-Weinberg equilibrium $(p<0.1)$. No significant differences among genotype frequencies were found with respect to age, $t(138)=0.703, p=0.48, \operatorname{sex}, t(138)=1.29 p=0.20$, or estimated IQ, $t(138)=0.35, p=0.72$.

Table 1 provides an overview of the outcomes for RTs and proportion of errors (PEs). RTs revealed a significant main effect of Trial Type, $F(1,138)=341.41 p<0.00001, \mathrm{MSE}=597.36$, $\eta^{2} p=0.71$; and of SOA, $F(4,552)=76.49 p<0.0001, \mathrm{MSE}=460.56$, $\eta^{2} p=0.36$. These two main effects were involved in two-way interaction, $F(1,138)=8.43 p<0.05$, MSE $=597.36, \eta^{2} p=0.06$, and in a three-way interaction involving DAT, $F(4,552)=3.01 p<0.05$, MSE $=290.38, \eta^{2} p=0.03$.

Both 10/10 homozygous and 9-repeat carriers showed a significant main effect of Trial Type, $F(1,57)=190.26, p<0.0001$, MSE $=612.659, \eta^{2} p=0.77$; and $F(1,81)=149.04, p<0.0001$, MSE $=586.599, \eta^{2} p=0.65$; respectively. However, $t$-tests comparing the magnitudes of the IOR effect revealed a significant difference between 10/10 homozygous and 9-repeat carriers in the shortest two SOAs (50 and $250 \mathrm{~ms}$ ), $t(138)=2.31, p=0.022$; and $t(138)=3.75, p=0.0001$, respectively, but not in the longer SOAs $(750,1000,1500 \mathrm{~ms}), t(138)=0.79, p=0.432 ; t(138)=1.40$, $p=0.16$; and $t(138)=0.08, p=0.933$. As obvious from Figure 2, 9-repeat and 10/10 homozygous carriers eventually reached the same maximum IOR, but the former reached it faster and earlier than the latter. 
Table 1 | Mean response latencies (in $\mathrm{ms}$ ), error rates (in percent), and loR effect (uncued - cued) for 9-repeat carriers and 10/10 Homozygous individuals.

\begin{tabular}{|c|c|c|c|c|c|c|c|c|c|c|}
\hline \multirow{2}{*}{$\frac{\text { Variables (SD) }}{\text { SOA }}$} & \multicolumn{5}{|c|}{ Nine-repeat carriers } & \multicolumn{5}{|c|}{ 10/10 Homozygous } \\
\hline & 50 & 250 & 750 & 1000 & 1500 & 50 & 250 & 750 & 1000 & 1500 \\
\hline \multicolumn{11}{|l|}{ CUED } \\
\hline $\mathrm{RT}(\mathrm{ms})$ & $375(5)$ & $366(6)$ & $370(6)$ & $361(6)$ & $357(6)$ & $375(4)$ & $361(5)$ & $375(5)$ & $366(5)$ & $364(5)$ \\
\hline Error rates (\%) & $0.4(0.2)$ & $1.5(0.4)$ & $2.6(0.5)$ & $3.3(0.5)$ & $3.2(0.5)$ & $0.2(0.2)$ & $1.2(0.3)$ & $2.2(0.4)$ & $2.4(0.4)$ & $2.0(0.4)$ \\
\hline \multicolumn{11}{|l|}{ UNCUED } \\
\hline $\mathrm{RT}(\mathrm{ms})$ & $367(6)$ & $336(6)$ & $328(6)$ & $322(6)$ & $335(6)$ & $380(5)$ & $349(5)$ & $336(5)$ & $332(5)$ & $341(5)$ \\
\hline Error rates (\%) & $0.3(0.1)$ & $1.3(0.3)$ & $2.7(0.4)$ & $3.7(0.5)$ & $2.6(0.5)$ & $0.1(0.1)$ & $1.0(0.2)$ & $2.3(0.4)$ & $2.5(0.4)$ & $2.9(0.4)$ \\
\hline \multicolumn{11}{|l|}{ IOR } \\
\hline $\mathrm{RT}(\mathrm{ms})$ & -8 & -30 & -42 & -38 & -22 & 5 & -12 & -39 & -34 & -23 \\
\hline Error rates (\%) & -0.1 & -0.2 & 0.1 & 0.4 & -0.6 & -0.1 & -0.2 & 0.1 & 0.1 & 0.9 \\
\hline
\end{tabular}

Significant group difference; ${ }^{*} p<0.05$. Standard errors in parentheses.

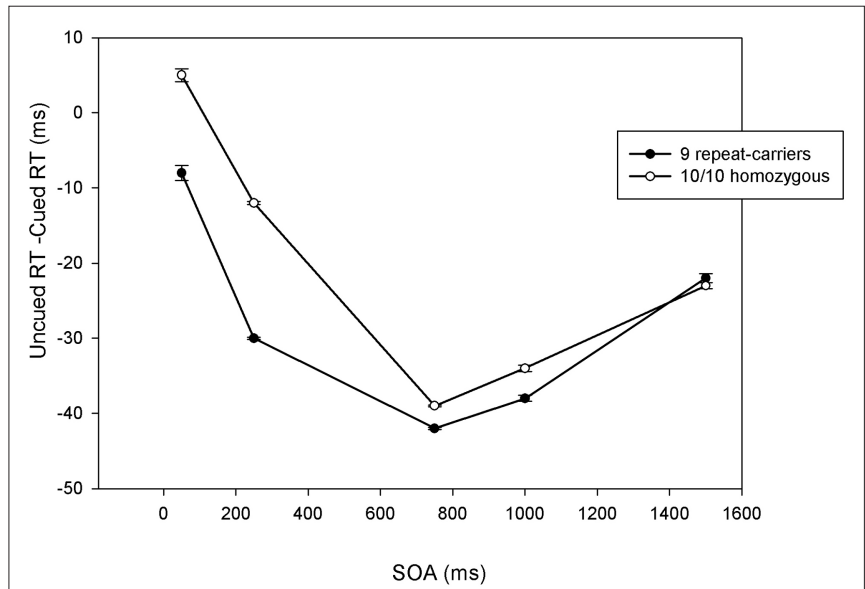

FIGURE 2 | Mean cueing effects (uncued reaction time, or RT, minus cued RT) at each stimulus-onset asynchrony (SOA) as a function of DAT polymorphism (10/10 homozygous vs. 9-repeat carriers). Positive numbers indicate facilitation; negative numbers indicate inhibition of return. Error bars indicate standard errors of the mean.

In the error analysis, SOA produced a main effect, $F(1,552)=13.87$, $p<0.0001, \mathrm{MSE}=38.167, \eta^{2} p=0.09$, due to fewer errors when the task was repeated than alternated. DAT1 was not involved in any significant effect.

\section{DISCUSSION}

Our findings provide evidence that DAT1, a gene coding the dopamine transporter (DAT) responsible of DA reuptake in the striatum (Sesack et al., 1998), modulates inhibitory input control, as indexed by IOR. Interestingly, 9-repeat carriers (with presumably higher striatal DA levels) showed an IOR that was comparable to that obtained in $10 / 10$ homozygous individuals with respect to its maximum size, but they reached it at shorter SOAs already. Apparently, then, DAT1 affects mainly the temporal dynamics of the interplay between facilitation and inhibition in the transition from cue-induced priming to the peak of IOR but not the maximum strength of inhibitory input control or the decay back towards baseline. In the absence of any hint to contributions from age, sex, or estimated IQ, we can rule out confounds with regard to these variables. In our case, the matching of the age range was of particular importance, as both inhibitory output control and IOR are known to be unrelated to general intelligence but sensitive to age (Logan, 1994; Castel et al., 2003; Langley et al., 2007).

Generally speaking, our observations are consistent with the assumption of a crucial role of dopaminergic pathways in IOR, as suggested by Poliakoff et al. (2003). They are also in line with patients and drug studies showing a reduced IOR in the case of striatal dopaminergic hypoactivity (Filoteo et al., 1997; Yamaguchi and Kobayashi, 1998; Couette et al., 2008; Colzato et al., 2009) and increased IOR in the case of enhanced striatal dopaminergic activity (Fillmore et al., 2005). More specifically, our findings provide support for the consideration of Cools (2008) and Cools and D'Esposito (2009) that higher striatal DA levels may promote cognitive flexibility. If we take the mechanism underlying IOR to bias the cognitive system towards the intake of novel information (Klein, 1988), our findings suggest that 9-repeat carriers of the DAT1 gene are biased towards flexibility rather than stability, at least as compared to 10-repeat carriers.

We can only speculate exactly how striatal DA levels manage to produce such a bias. There is strong, uncontroversial evidence for a crucial role of the superior colliculus in the emergence of IOR. One example is the study of Dorris et al. (2002), who used singlecell recordings to investigate the neural responses in the superior colliculus of the monkey. Neural responses to the target stimulus were reduced if it was preceded by a cue in the same location, and the amount of reduction was correlated with the behavioral IOR effect. However, the neurons coding for the cue were no less but, if anything, more active after the cue presentation, suggesting that the reduced response to the target was not simply a result of the integration of cue- and target-related activation. In other words, IOR is reflected in the neural response of the superior colliculus but it is not created there.

A series of pharmacological single-cell studies by Hikosaka and Wurtz (1985a,b) suggests one possible origin. Hikosaka and Wurtz (1985a) provided evidence that regional activation of the superior colliculus is controlled by collicular GABA neurons, that hold the system in (inhibitory) check and allow for saccades and 
attention shifts by means of selective disinhibition. Hikosaka and Wurtz (1985b) went on to demonstrate that these collicular GABA neurons are controlled by the substantia nigra. Our finding of a more pronounced IOR in 9-repeat than in 10-repeat carriers fits nicely into this picture. It has been assumed that the presumably lower striatal dopamine level associated with the DAT 10-repeat allele may lead to reduced DAD2 signaling and increased GABA release (cf., Seamans and Yang, 2004). This could be expected to act like a GABA agonist on the substantia nigra, which in turn would reduce the inhibitory control exerted on the superior colliculus and, thus, facilitate activation there. Accordingly, the coding of the target in the superior colliculus would evoke relatively stronger neural responses in 10-repeat than in 9-repeat carriers and, thus, reduce the IOR exhibited by the former - just as we have observed.

At this point this scenario is no more than speculation and this cannot be the whole story. It is logically possible that IOR results from the dynamics of the substantia nigra itself: the temporal disinhibition necessary to release activation in the superior colliculus may be achieved by a phasic inhibition of collicular GABA neurons, which automatically go back to their base level after some time. However, the characteristics of IOR are task dependent (Fecteau and Munoz, 2003), as are its temporal dynamics (Lupianez et al., 2001). Indeed, researchers have championed the frontal eye fields (e.g., Ro et al., 2003) and the posterior parietal cortex (e.g., Dorris et al., 2002) as important contributors to the control of the superior colliculus. Interestingly, both of these areas are interfaced to the colliculus via the substantia nigra, even though direct connections also exist (see Schiller and Tehovnik, 2005). It is thus possible that the substantia nigra serves as an integrator of different control signals, which together modulate the neural response of the colliculus to external stimulation. The integrated signal would not necessarily create, or be responsible for the occurrence of IOR as such, but it might be functional in delaying or even preventing IOR altogether whenever needed.

What is clear from our findings, however, is that the DAT1 gene, and the neuromodular processes depending on it, play an important role in the emergence and behavioral expression of IOR. Presumably, they do so by modulating the reciprocal interplay between prefrontal and striatal networks in an attempt to achieve the proper balance between cognitive stability and flexibility.

\section{ACKNOWLEDGMENTS}

We thank Sabine Maaskant, Willem Turnhout, Stephanie Greve, Raoul Putman, Laura Rus, Alain Boersen, Marieke van der Meer, Linda van Hooidonk, Martine van Nierop, and Doris van Dijk for their enthusiasm and invaluable assistance in recruiting, testing the participants of this study, and collecting the data. We would also like to thank Ruo Mu Guo for programming the experiment.

\section{FUNDING}

The research of Lorenza S. Colzato is supported by NWO (Netherlands Organization for Scientific Research).

\section{REFERENCES}

Bertolino, A., Blasi, G., Latorre, V., Rubino, V., Rampino, A., Sinibaldi, L., Caforio, G., Petruzzella, V., Pizzuti, A., Scarabino, T., Nardini, M., Weinberger, D. R., and Dallapiccola, B. (2006). Additive effects of genetic variation in dopamine regulating genes on working memory cortical activity in human brain. J. Neurosci. 26, 3918-3922.

Castel, A., Chasteen, A. L., Scialfa, C. T., and Pratt, J. (2003). Adult age differences in the time course of inhibition of return. J. Gerontol.: Psychol. Sci. 58, 256-259.

Colzato, L. S., and Hommel, B. (2009). Recreational use of cocaine eliminates inhibition of return. Neuropsychology 23, 125-129.

Colzato, L. S., Waszack, F. Nieuwenhuis, S. T., Posthuma, D., and Hommel, B. (2010). The flexible mind is associated with the Catechol-O-methyltransferase (COMT) Val158Met polymorphism: evidence for a role of dopamine in the control of task switching. Neuropsychologia. doi: 10.1016/j. neuropsychologia.2010.04.023.

Cools, R. (2008). Role of dopamine in the motivational and cognitive control of behaviour. Neuroscientist 14, 381-395.

Cools, R., and D'Esposito, M. (2009). "Dopaminergic modulation of flex- ible cognitive control in humans," in Dopamine Handbook. eds A. Björklund, S. Dunnett, L. Iversen, and S. Iversen (Oxford: Oxford University Press), pp 249-260.

Couette, M., Bachoud-Levi, A. C., Brugieres, P., Sieroff, E., and Bartolomeo, P. (2008). Orienting of spatial attention in Huntington's disease. Neuropsychologia 46, 1391-400.

Dickinson, S. D., Sabeti, J., Larson, G. A., Giardina, K., Rubinstein, M., Kelly, M. A., Grandy, D. K., Low, M. J., Gerhardt, G. A., and Zahniser, N. R. (1999). Dopamine D2 receptor-deficient mice exhibit decreased dopamine transporter function but no changes in dopamine release in dorsal striatum. J. Neurochem. 72, 148-156.

DNA Genotek, Inc. (2006). OrageneTM Product Brochure. Ottawa: DNA Genotek, Inc.

Dorris, M. C., Klein, R. M., Everling, S., and Munoz, D.P. (2002). Contribution of the primate superior colliculus to inhibition of return. J. Cogn. Neurosci. 14, 1256-1263.

Elzinga, B. M., Ardon, A. M., Heijnis, M. K., de Ruiter, M. B., van Dyck, R., and Veltman, D.J. (2007). Neural correlates of enhanced working-memory performance in dissociative disorder: a functional MRI study. Psychol. Med. $37,235-245$.
Elzinga, B. M., Roelofs, K., Tollenaar, M. S., Bakvis, P., van Pelt, J., and Spinhoven, P. (2008). Diminished cortisol responses to psychosocial stress associated with lifetime adverse events: a study among healthy young subjects. Psychoneuroendocrinology 33 , 227-237.

Fecteau, J. H., and Munoz, D. P. (2005). Correlates of raphne of attention and inhibition of return across stages of visual processing. J. Cogn. Neurosci. 17, 1714-1727.

Fecteau, J. H., and Munoz, D. P. (2003). Exploring the consequences of the previous trial. Nat. Rev. Neurosci. 4 435-443.

Fillmore, M. T., Rush, C. R., and Abroms, B. D. (2005). d-Amphetamineinduced enhancement of inhibitory mechanisms involved in visual search. Exp. Clin. Psychopharmacol. 13, 200-208.

Filoteo, J. V., Delis, D. C., Salmon, D. P., Demadura, T., Roman, M. J., and Shults, C. W. (1997). An examination of the nature of attentional deficits in patients with Parkinson's disease: evidence from a spatial orienting task. J. Int. Neuropsychol. Soc. 3, 337-347.

Giros, B., Mestikawy, S., Godinot, N., Zheng, K., Han, H., Yang-Feng, T., Caron, M. G. (1992). Cloning, pharmacological characterization, and chromosome assignment of the human dopamine transporter. Mol. Pharmacol. 42, 383-390.

Goschke, T. (2000). “Involuntary persistence and intentional reconfiguration in task-set switching," in Attention and performance XVIII: control of Cognitive Processes, eds S. Monsell and J. Driver (Cambridge, MA: MIT Press), 331-355.

Hikosaka, O., and Wurtz, R. H. (1985a). Modification of saccadic eye movements by GABA-related substances. I. Effect of muscimol and bicuculline in monkey superior colliculus. $J$. Neurophysiol. 53, 266-291.

Hikosaka, O., and Wurtz, R. H. (1985b). Modification of saccadic eye movements by GABA-related substances. II. Effects of muscimol in the monkey substantia nigra. J. Neurophysiol. 53, 292-308.

Kang, A. M., Palmatier, M. A., and Kidd, K. K. (1999). Global variation of a 40-bp VNTR in the 30-untranslated region of the dopamine transporter gene (SLC6A3). Biol. Psychiatry 46, 151-160.

Karoum, F., Chrapusta, S. J., and Egan, M. F. (1994). 3-Methoxytyramine is the major metabolite of released dopamine in the rat frontal cortex: reassessment of the effects of antipsychotics on the dynamics 
of dopamine release and metabolism in the frontal cortex, nucleus accumbens, and striatum by a simple two pool model. J. Neurochem. 63, 972-979.

Klein, R. M. (1988). Inhibitory tagging system facilitates visual search. Nature 334, 430-431.

Langley, L. K., Fuentes, L. J., Vivas, A. B., and Saville, A. L. (2007). Aging and temporal patterns of inhibition of return. J. Gerontol. B: Psychol. Sci. Soc. Sci. 62, 71-77.

Lecrubier, Y., Sheehan, D. V., Weiller, E., Amorim, P., Bonara, I., Sheehan, K., Janavs, J., Dunbar, G. (1997). The mini international neuropsychiatric interview (MINI). A short diagnostic structured interview: reliability and validity according to the CIDI. Eur. Psychiatry 12, 224-231.

Logan, G. D. (1994). "On the ability to inhibit thought and action: a users' guide to the stop signal paradigm," in Inhibitory Processes in Attention, Memory and Language, eds D. Dagenbach and T.H. Carr (San Diego: Academic Press), 189-239.

Lupianez, J., Decaix, C., Sieroff, B., Milliken, B., and Bartolomeo, P. (2004). Independent effects of endogenous and exogenous spatial cueing: Inhibition of return for endogenously attended target locations. Exp. Brain Res. 159, 447-457.

Lupianez, J., Milliken, B., Solano, C., Weaver, B., and Tipper, S. P. (2001). On the strategic modulation of the time course of facilitation and inhi- bition of return. Q. J. Exp. Psychol. 54A, 753-773.

Meiergerd, S. M., Patterson, T. A., and Schenk, J. O. (1993). D2 receptors may modulate the function of the striatal transporter for dopamine: kinetic evidence from studies in vitro and in vivo. J. Neurochem. 61, 764-767.

Mitchell, R. J., Howlett, S., Earl, L., White, N. G., Crawford, M. H., McComb, J., Schanfield, M. S., Briceno, I., Papiha, S. S., Osipova, L., Livshits, G., Leonard, W. R., and Crawford, M. H. (2000). The distribution of the 3'VNTR polymorphism in the human dopamine transporter gene in world populations. Hum. Biol. 72, 295-304.

Mortensen, O.V., and Amara, S. G. (2003). Dynamic regulation of the dopamine transporter. Eur. J. Pharmacol. 479, 159-170.

Poliakoff, E., O’Boyle, D. J., Moore, A. P., McGlone, F. P., Cody, F. W. J., and Spence, C. (2003). Orienting of attention and Parkinson's disease: tactile inhibition of return and response inhibition. Brain 126, 2081-2092.

Posner, M. I., and Cohen, Y. (1984). "Components of visual orienting," in Attention and Performance X: Control of Language Processes, eds H. Bouma and D. G. Bouwhuis (Hillsdale, NJ: Erlbaum), 531-556.

Raven, J. C., Court, J. H., and Raven, J. (1988). Manual for Raven's progressive matrices and vocabulary scales. London: Lewis.

Ro, T., Farné, A., and Chang, E. (2003). Inhibition of return and the human frontal eye fields. Exp. Brain Res. 150, 290-296.

Schiller, P. H., and Tehovnik, E. J. (2005) Neural mechanisms underlying target selection with saccadic eye movements. Prog. Brain Res. 149, 157-171.

Seamans, J. K., Yang, C. R. (2004). The principal features and mechanisms of dopamine modulation in the prefrontal cortex. Prog. Neurobiol. 74, 1-58.

Sesack, S. R., Hawrylak, V. A., Matus, C., Guido, M. A., and Levey, A. I. (1998). Dopamine axon varicosities in the prelimbic division of the rat prefrontal cortex exhibit sparse immunoreactivity for the dopamine transporter. $J$. Neurosci. 18, 2697-2708.

Sheehan, D. V., Lecrubier, Y., Sheenan, K. H., Amorim, P., Janavs, J., Weiller, E., Hergueta, T., Baker, R., and Dunbar, G. C. (1998). The mini-international neuropsychiatric interview (M.I.N.I.): the development and validation of a structured diagnostic psychiatric interview for DSM-IV and ICD-10. J. Clin. Psychiatry 59, 22-23.

Vandenbergh, D. J., Persico, A. M. Hawkins, A. L., Griffin, C. A., Li, X., Jabs, E. W. et al. (1992). Human dopamine transporter gene maps to chromosome 5p15.3 and displays a VNTR. Genomics 14, 1104-1106.

Volkow, N. D., Fowler, J. S., and Wang, G. J. (1999). Imaging studies on the role of dopamine in cocaine reinforcement and addiction in humans. $J$. Psychopharmacol. 13, 337-345.
Waldman, I. D., Rowe, D. C. Abrinteamowitz, S., Kozel, T., Mohr, J. H., Sherman, L. et al. (1998). Association and linkage of the dopamine transporter gene and attention-deficit hyperactivity disorder in children: heterogeneity owing to diagnostic subtype and severity. Am. J. Hum. Genet. 63, 1767-1776.

Yamaguchi, S., and Kobayashi, S. (1998). Contributions of the dopaminergic system to voluntary and automatic orienting of visuospatial attention. J. Neurosci. 18, 1869-1878.

Conflict of Interest Statement: The authors declare that the research was conducted in the absence of any commercial or financial relationships that could be construed as a potential conflict of interest.

Received: 17 March 2010; paper pending published: 12 May 2010; accepted: 07 June 2010; published online: 13 July 2010. Citation: Colzato LS, Pratt J and Hommel B (2010) Dopaminergic control of attentional flexibility: inhibition of return is associated with the dopamine transporter gene (DAT1). Front. Hum. Neurosci. 4:53. doi: 10.3389/fnhum.2010.00053

Copyright (c) 2010 Colzato, Pratt and Hommel. This is an open-access article subject to an exclusive license agreement between the authors and the Frontiers Research Foundation, which permits unrestricted use, distribution, and reproduction in any medium, provided the original authors and source are credited. 